\title{
Resonant Metagratings for Spectral and Angular Control of Light for Colored Rooftop Photovoltaics
}

\author{
Floris Uleman, Verena Neder,* Andrea Cordaro, Andrea Alù, and Albert Polman \\ Cite This: ACS Appl. Energy Mater. 2020, 3, 3150-3156 \\ Read Online
}

ABSTRACT: We designed semitransparent metagrating supercells that enable control over the spectrum and directivity of incident light for applications in photovoltaics with tailored angular appearance. The building block of the supercells is a 100-120 nm wide and $175 \mathrm{~nm}$ tall silicon nanowire that shows a strong Mie resonance around $\lambda=650 \mathrm{~nm}$. By arranging the resonant Mie scatterers into metagratings of increasing pitch (675-1300 nm), we created a Lambertian-like scattering distribution over an angular range of choice. The millimeter-sized metasurfaces were fabricated using electron beam lithography and reactive ion etching. The fabricated metasurface nearly fully suppresses specular reflection on resonance while $10 \%$ of the incoming light around the resonance is scattered into the angular range between $30^{\circ}$ and $75^{\circ}$, creating a bright red appearance over this specific range of angles. Off-resonant light in the blue, green, and near-

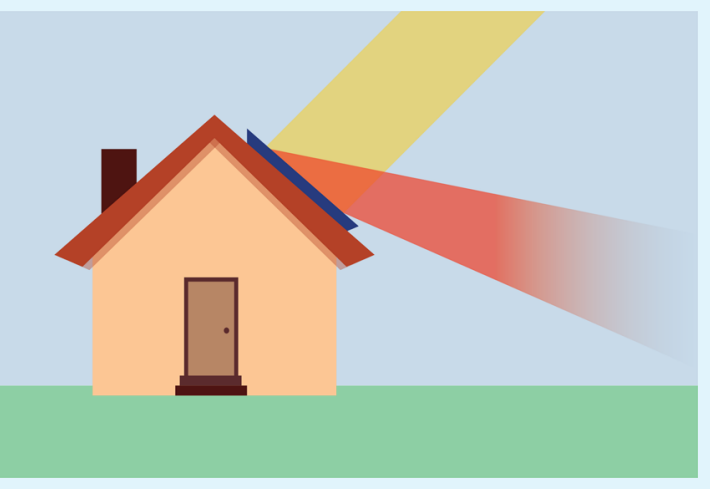
infrared is efficiently transmitted through the metasurface and absorbed in the underlying photovoltaic cell. The implemented silicon heterojunction solar cells with integrated metagrating supercells shows a reduction in external quantum efficiency matching the resonant scattering spectral range. The short circuit current is reduced by $13 \%$ due to the combined effects of resonant scattering, reflection from the high-index substrate, and absorption in the Si nanowires. In addition to efficient colorful photovoltaics with tailored angular appearance, the metagrating concept can find application in many other light management designs for photovoltaics and other optoelectronic devices.

KEYWORDS: colored photovoltaics, BIPV, transparent metagratings, metasurface, light management, solar cells, silicon nanoparticles, Mie resonators

\section{INTRODUCTION}

New solar power conversion architectures are increasingly important to achieve large-scale sustainable power generation to sustain the growing energy needs of our society. Integration of photovoltaics (PVs) into the local architecture, buildings, and urban terrains is a rapidly growing research and technology area and will be crucial to meet the requirements set by the Paris agreement in the coming years. ${ }^{1}$ Current commercial solar cells and modules typically have a blue or black color that does not always fit within the urban design. Modules with tailored color are necessary to integrate PVs into residential and modern city building architecture, where aesthetics plays a key role. In order to achieve both functional beauty and high power conversion efficiency, it is essential to develop innovative PV solutions to create colored solar cells while maintaining high efficiencies.

Over the past decades, research has shown different approaches to achieve colored PVs. Interference coatings and Bragg reflectors have been used to modify the reflected spectrum but do not offer control over the angular scattering. ${ }^{2-6}$ Colored optical coatings, based on polymer or luminescent materials, have been applied on the front module glass of cells $\mathrm{s}^{7-10}$ but are often limited by absorption in the coating material. Other solutions, specifically for solar roof tiles, have been offered to control the field of view with louver films that shade the cells on a rooftop for observers from the ground. ${ }^{11}$ This simple approach shows esthetical results; however, the performance of the solar cell is highly restricted if the sun shines at a low angle, because the louver film also shades the sunlight. Scattering from plasmonic nanostructures has been applied in colorful solar cells but suffers from fundamental optical losses in the metal. ${ }^{12,13}$ Similarly, resonant dielectric nanoparticles have been used for spectrally controlled light scattering with minimal optical losses. ${ }^{14-18}$ Recently, these nanostructures have been employed for the creation of colorful solar cells, with the advantage of very little loss and scalable fabrication possibilities. ${ }^{19-21}$

Received: January 7, 2020

Accepted: April 3, 2020

Published: April 3, 2020 
a)

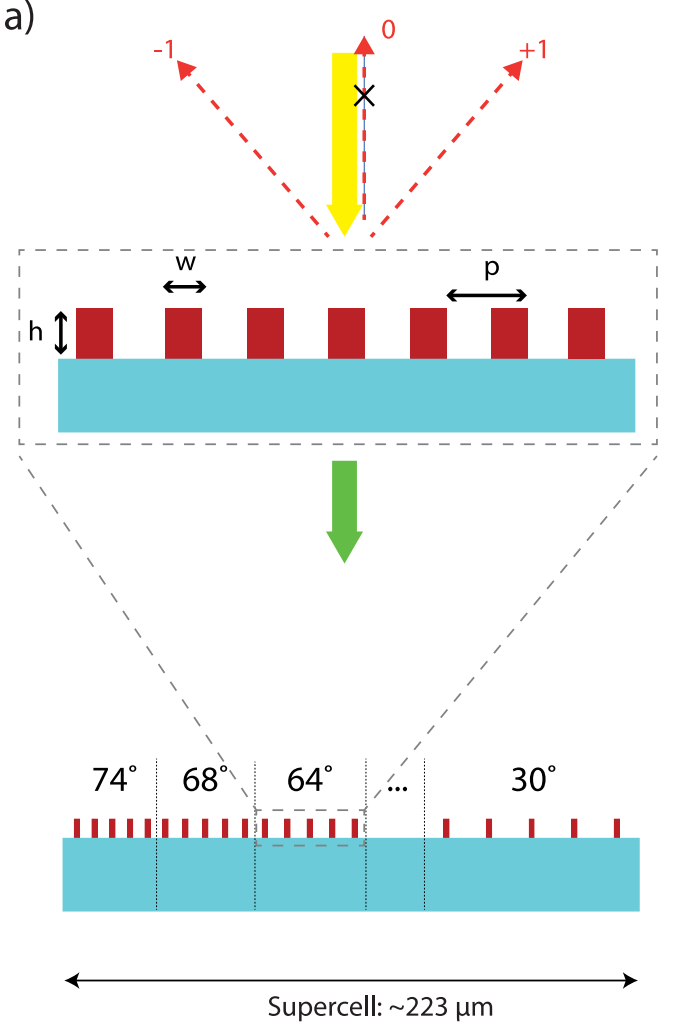

b)

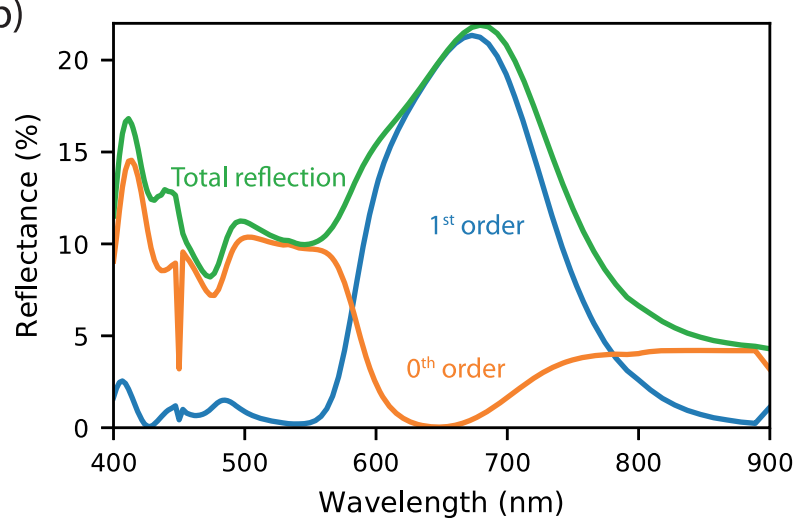

c)

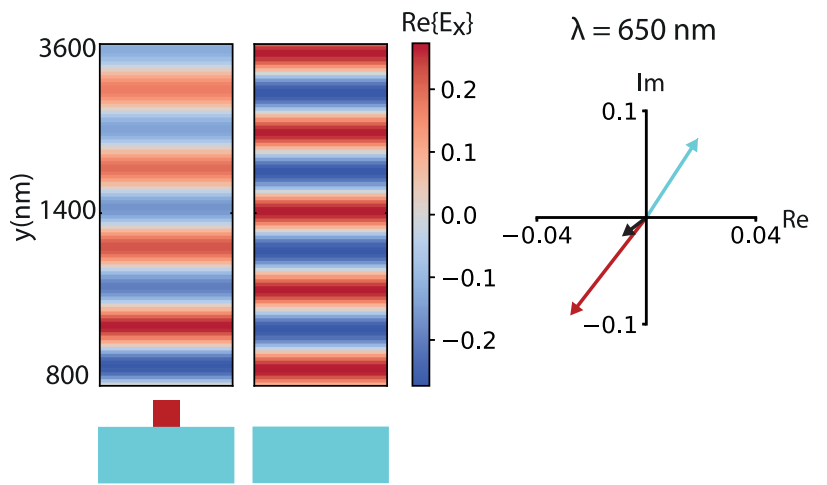

Figure 1. Principle of transparent metagrating. (a) Top: Schematic overview of a transparent metagrating. Mie silicon nanowire resonators are placed periodically on top of a transparent substrate. The light around the resonance is scattered into diffraction orders. Bottom: Schematic overview of the complete supercell design. There are 25 metagratings placed next to each other to form a supercell that reflects light around the resonance wavelength toward an angle range of $30-75^{\circ}$. (b) Simulated reflectance of a metagrating of silicon resonators $(w=110 \mathrm{~nm}, h=175 \mathrm{~nm}$, $p=900 \mathrm{~nm}$ ). The total reflectance (green line), specular reflectance (orange line), and diffraction into the first $(+1$ and -1$)$ order (blue line) are plotted. (c) Scattered E field radiated upward from the single nanowire (left) and field reflected off the sapphire substrate (right) at the wavelength of cancellation $\lambda=650 \mathrm{~nm}$. Phasor representation of the scattered $\mathbf{E}$ field at a position in the upward scattering direction from the nanowire array (red arrow) and the substrate (light blue arrow), and their sum (black arrow).

So far, few of the proposed designs for colored PVs exhibit both angular and spectral control on the reflected light. The possibility of designing the scattering angular profile at will is particularly appealing in colored PV applications for two main reasons: First, for a colored surface to appear matte, its angular reflectance has to follow the Lambertian cosine law (i.e., the apparent brightness of the scattered light should be the same for an observer from every angle). Second, it is important to reflect the color to a specific field of view of choice in order to minimize the losses as much as possible. The most obvious example is the application of directional colored solar cells on rooftops, where a red color appearance should be seen only from an observer near the building, while reflection upward is undesired.

The use of scattering dielectric nanoparticles allows controlling not only the spectrum, by exciting Mie resonances, but also the directivity by smartly arranging the scatterers into a metasurface. In previous studies, different metasurfaces, specifically metagratings, have been introduced to allow directional control of resonant reflection. ${ }^{22-29}$

In this work, we demonstrate how the geometry of an array of dielectric Mie scatterers arranged in a grating structure can be tailored such that light in a narrow spectral band is scattered over a well-defined angular range. To this end, we designed a supercell of multiple metagratings consisting of dielectric nanoparticles that show Lambertian resonant scattering while the nonresonant spectral bands are effectively transmitted. We demonstrate these concepts in a one-dimensional geometry employing Mie resonances in subwavelength silicon nanowires (NWs) that allow us to gain spectral control over the reflected light for one polarization direction; the design can be readily expanded to two dimensions.

The scattering resonances are tuned by optimizing the shape and spatial dimensions of the nanowires such that the scattering profile matches the desired scattering spectrum. We demonstrate resonant scattering around $\lambda=650 \mathrm{~nm}$, yielding a red scattering profile. Next, the NWs are placed in multiple gratings on a transparent substrate that scatter light with a tailored distribution over the first diffraction orders. We apply the metasurface onto a Si solar cell and demonstrate that effective light scattering in a narrow spectral and angular band is combined with high external quantum efficiency of the solar cell.

\section{THEORY AND DESIGN}

In this section, we introduce the concept of a Mie-resonant metagrating and outline how to leverage its properties to tailor at will the spectral and angular response of the metasurface. Specifically, for rooftop PV applications, we design the response to correspond to a Lambertian angular profile spectrally peaked in the red portion of the visible spectrum. Different from previous approaches, ${ }^{29}$ the Mie resonators are placed on a transparent substrate such that only the resonant 
portion of the spectrum is reflected, while the transmitted light can be conveniently collected by a solar cell (see Figure 1a).

Upon excitation of Mie resonances, high-index dielectric nanoantennas can strongly scatter the incoming light. ${ }^{15,30,31}$ In particular, here we exploit the modes sustained by NWs made of silicon, taking advantage of its high refractive index. The scatterers are Si NWs of height $h=175 \mathrm{~nm}$ and width $w=$ $100-120 \mathrm{~nm}$ on a sapphire substrate. The resonant wavelength of modes supported by such a high-index scatterer can easily be tuned by changing the size of the scatterer, thus enabling spectral control. Details about the scattering cross section of the single $\mathrm{Si}$ NW are given in the SI. In this work, the metasurface is designed to scatter light within a narrow bandwidth around $\lambda=650 \mathrm{~nm}$.

Next, the Mie scatterers are placed into an array with periodicity $p$, such that first-order diffraction in air occurs while the second order is still evanescent. This is the case for periodicities in the range $650 \mathrm{~nm}<p<1300 \mathrm{~nm}$, according to the grating equation for normally incident light $p \sin \theta_{m}=m \lambda$, where $\theta_{m}$ is the angle at which light is diffracted, $m$ is an integer denoting the order of diffraction, and $\lambda$ is the wavelength of the incident light. In this work, as light is normally incident on the grating, two symmetric first $(+1$ and -1$)$ diffraction orders exist that we refer to as first orders from now on. Figure $1 \mathrm{~b}$ shows the simulated ${ }^{32,33}$ reflectance spectrum of a single grating with pitch $p=900 \mathrm{~nm}$, where the unit cell is composed of a single Si NW ( $w=110 \mathrm{~nm}, h=175 \mathrm{~nm})$ on sapphire, and it is illuminated at normal incidence by a plane wave polarized perpendicular to the NW (TE polarized). The reflectance into the zeroth and the \pm first diffraction orders $\left(\theta_{1}= \pm 46.2^{\circ}\right.$ at $\lambda=$ $650 \mathrm{~nm})$ reveals three important features. First, a clear peak around the design resonance $\lambda=650 \mathrm{~nm}(\sim 20 \%)$ is observed due to resonant excitation of the modes of the Si NWs around this wavelength. Second, another peak around $\lambda=410 \mathrm{~nm}$ is observed $(\sim 15 \%)$, originating from a higher-order mode excitation. Third, a significant portion of the green and blue spectral range is directly reflected off the sapphire substrate to the zeroth order $(\sim 10 \%)$. It is important to note that most of the reflected light around the resonance is scattered into the first orders $(\sim 20 \%)$, while reflection back to the zeroth order is suppressed $(\sim 2 \%)$. In order to investigate the physical mechanism behind the suppression of specular reflection, we use the total-field-scattered-field method. ${ }^{34}$ Light resonantly scattered by the NWs interferes destructively with light reflected off the bare sapphire substrate, hence resulting in a suppressed reflection to the zeroth order. For destructive interference to arise, the amplitude of the reflected light from the substrate and that scattered by the NWs is designed to be equal, while the phase is shifted by $\pi$. This cancellation phenomenon is outlined in Figure $1 c$ by investigating the scattered fields of the two scenarios. The field scattered by a single NW (left) and the one reflected by the sapphire substrate (right) are shown, when illuminated at normal incidence by a plane wave at $\lambda=650 \mathrm{~nm}$ (TE). In the first case, the total-field-scattered-field approach allows one to look only at the scattering contribution of the NW, and not of the substrate. Importantly, only the upward scattering in the specular direction is shown here. The outgoing specular reflected field from the bare sapphire substrate shows a clear phase shift compared to the scattered field of the NW. In addition, the phasor representation at a position in the upward scattering direction of the scattered (NWs) and reflected (bare sapphire) fields is shown for $\lambda=650 \mathrm{~nm}$. In the complex plane, the electric field of the scattered light is depicted for both scenarios and allows a precise comparison of the amplitude (length of arrow) and phase (angle in complex plane). For the NW scenario, the amplitude was taken from the scattered wavefront of an array of NWs (pitch $p=900 \mathrm{~nm}$ ), as the amplitude of a single NW is decreasing radially. The phase of the scattered field in the backward direction is equal for a single NW or a NW array. Notably, it can be seen that the phase shift is almost $\pi$ while the amplitude is close to equal at the wavelength of cancellation. The sum of the two vectors (black vector) shows that the cancellation is close to ideal. The reflection from the substrate is thus fundamental to achieve the cancellation in specular direction as discussed in more detail in the Supporting Information and also explained in ref 29.

By considering different pitches, each targeting a different outgoing angle of diffraction, and suitably combining them into a larger supercell, it is possible to design a prescribed overall angular response. A schematic overview of the design, resulting in a supercell that scatters from $30^{\circ}$ to $75^{\circ}$ for $\lambda=650 \mathrm{~nm}$ (pitch: 675-1300 nm), is presented in the bottom of Figure 1 a.

In colored PV applications, it is important that a Lambertian reflected angular profile is achieved to perceive a matte appearance. ${ }^{19}$ However, in rooftop PV applications, this requirement can be relaxed since the metasurface should appear Lambertian red only from an observer on the street. Hence, assuming that the sun is incident normal to the solar cells on a rooftop, we can disregard small diffracted angles in the design. Since common house roofs have an angle of $\sim 40^{\circ}$, the desired angular span of the designed angular response lies in the range $30^{\circ}-75^{\circ}$. Indeed, sunlight that hits the panel perpendicular is scattered toward the field of view of observers on the street. As mentioned before, the overall structure is realized by placing multiple metagratings next to each other, creating a "supercell" array of silicon nanowires with varying periodicities (see Figure 1a). Within this procedure, it is also important to take into account the relative area of each metagrating (adjusted by their own efficiencies) in order to properly reproduce the Lambertian intensity distribution in reflection. The total area of the supercell is the sum of all grating areas, and it adds up to $223 \mu \mathrm{m}$. The specific values of $p, w$, the total reflection and reflection to the first order for individual metagratings, and the number of unit cells used in each of the metagratings composing the supercell are summarized in Table S1.

\section{EXPERIMENTS}

In order to experimentally demonstrate the possibility of gaining angular and spectral control via transparent metagratings, we fabricated the structure described above and characterized it optically. Next, we compare the measured data to numerical simulations and assess the performance of a solar cell with the optimized metasurface integrated into its structure.

3.1. Fabrication. The metagratings were fabricated using electron beam lithography (EBL), as described in the following. First, siliconon-sapphire substrates $(500 \mathrm{~nm} \mathrm{c-Si}$ on $0.46 \mathrm{~mm}$ R-plane sapphire, MTI Corporation) were cleaned in base piranha. The silicon layer is etched down to a thickness of $175 \mathrm{~nm}$ using reactive ion etching (RIE) using a $\mathrm{CHF}_{3}(15 \mathrm{sccm})-\mathrm{SF}_{6}(10 \mathrm{sccm})-\mathrm{O}_{2}(3 \mathrm{sccm})$ plasma etch. Next, a 50-60 nm HSQ high-resolution negative resist layer was spin-coated on the sample and baked for $150 \mathrm{~s}$ at $180{ }^{\circ} \mathrm{C}$. For better conductivity, Electra 92 (AR-PC-5091) was spin-coated on the sample. The substrate was then exposed using a Raith Voyager lithography system (dose of $2.400 \mu \mathrm{C} / \mathrm{cm}^{2}$ ). To obtain a sample that 
is large enough for optical measurements, the supercell of $223 \mu \mathrm{m}$ described above is replicated 6 times next to each other, resulting in a $1.35 \mathrm{~mm}$ wide field. After e-beam writing, the sample was developed in TMAH for $75 \mathrm{~s}$ at $50{ }^{\circ} \mathrm{C}$. The patterned HSQ is then used as a hard mask to structure the underlying $\mathrm{Si}$ layer using two subsequent RIE etching steps. First, the native oxide of the silicon is etched using $\mathrm{Cl}_{2}(60 \mathrm{sccm})$, and then the silicon is etched in a $\mathrm{HBr}(48.6 \mathrm{sccm})$ and $\mathrm{O}_{2}(1.4 \mathrm{sccm})$ plasma at $60^{\circ} \mathrm{C}$.

The result of the described fabrication is shown in Figure 2. The sample consists of silicon NWs with a remaining HSQ layer on top,

a)

C)
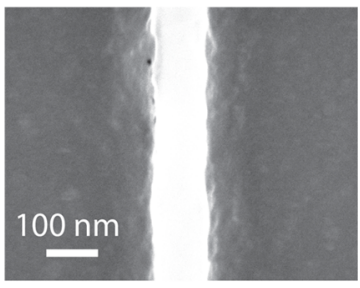

b)

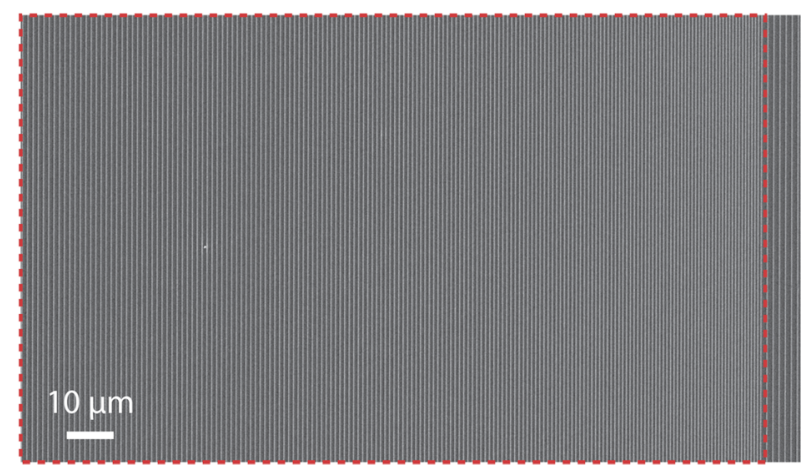

Figure 2. SEM image of fabricated metasurface. (a) Top-view SEM of a single silicon nanowire of $w=120 \mathrm{~nm}$ on top of sapphire substrate. (b) Tilted image of a single silicon nanowire (tilt angle $45^{\circ}$ ). (c) Topview of full supercell (dashed red line) containing all metagratings with varying pitch $(p=675-1300 \mathrm{~nm})$. Stitching of two supercells can be seen on the right side of the image.

on a sapphire substrate. A single NW is depicted in Figure 2a,b, showing that the side walls are etched perpendicular to the substrate. The HSQ on top of the nanowire is not removed as this process involves chemical reactions that also react with the sapphire substrate. For better resolution, a layer of Electra 92 (AR-PC-5091) was spincoated on the sample before imaging. An overview SEM image of the total supercell is shown in Figure 2c. The pitch between the wires is decreasing from left $(p=1300 \mathrm{~nm})$ to right $(p=675 \mathrm{~nm})$, and for the complete area of the supercell, a good uniformity was achieved. On the right side of the supercell, the beginning of the next identical supercell is visible.

3.2. Optical Characterization. To distinguish light reflected specularly ( $0^{\text {th }}$ order) from light reflected at an angle ( $1^{\text {st }}$ orders), we use an integrating sphere. We measure both the sample's total reflectance and the total reflectance excluding the zeroth order. The difference between the two measurements then yields the zerothorder reflection (see Supporting Information for a schematic of the setup).

Figure $3 \mathrm{a}$ shows the measured specular reflectance spectrum, as well as the total reflectance spectrum excluding the specular contribution, which we refer to as the scattered spectrum. The latter shows a broad peak centered at $\lambda=614 \mathrm{~nm}$ reaching $9.9 \%$ reflectance. The full width at half-maximum of this peak is around $200 \mathrm{~nm}$, covering the wavelength range of interest for red color perception. At the same time, in the same bandwidth around $\lambda=614 \mathrm{~nm}$, the zerothorder reflection is strongly suppressed, as desired. Indeed, the zeroth-

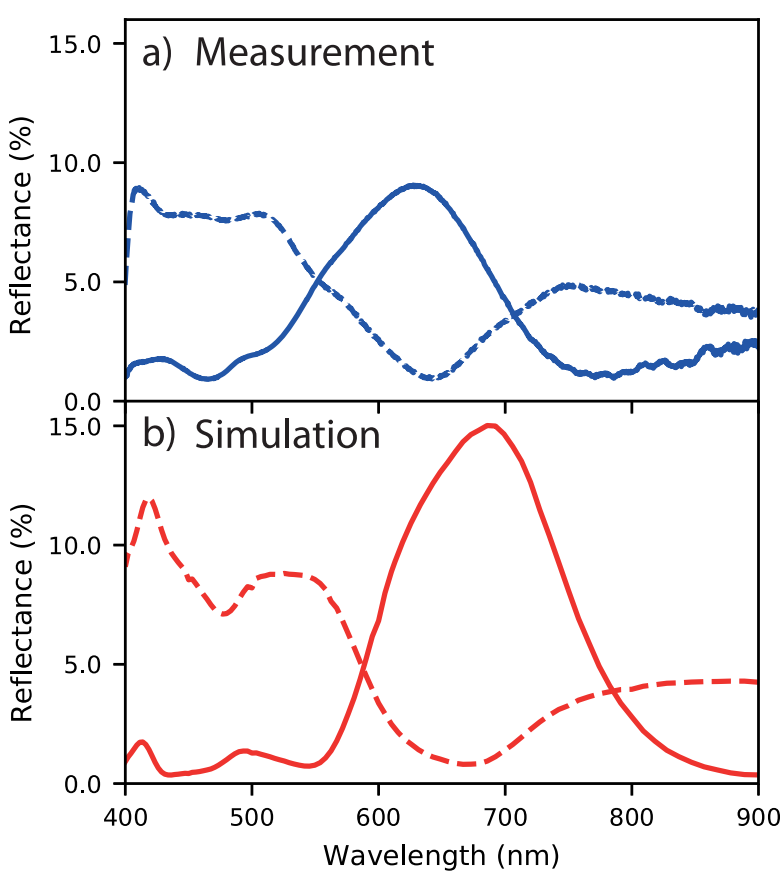

Figure 3. Reflectance spectra of full metasurface. (a) Measured specular reflectance spectrum (dashed blue line) and scattered spectrum (solid blue line) of full metasurface. (b) Simulated specular reflectance (dashed red line) and reflectance for diffraction order (solid red line) of a single supercell. Reflectance is determined by the contribution of the different metagratings in the supercell, weighted by their area, respectively.

order reflectance is around $8 \%$ in the blue wavelength range and decreases to $2 \%$ on resonance demonstrating efficient light redirection. Additionally, the reflection at wavelengths above the resonance is slightly decreased.

Figure $3 \mathrm{~b}$ shows simulated scattered and specular reflectance spectra. The experimentally measured trends show good agreement with the simulated spectra: a broad peak around $680 \mathrm{~nm}$ in the averaged first-order reflection (average reflectance to first orders: sum of the reflectance from the different metagratings composing the metasurface, weighted by their relative area) corresponds to a clear reduction in specular reflection. The peak position can be explained by the combined contributions of the different metagratings (see SI). We ascribe the lower measured reflectance compared to the simulations to fabrication imperfections resulting in silicon NWs with slightly different dimensions, as well as small slanting of the sidewalls which results in a less pronounced resonance. ${ }^{35}$

Figure 4a shows the angular reflectance as a function of wavelength, measured using a rotating stage setup (see ref 29 for the description of measurement setup). The measurements were performed in the wavelength range $\lambda=550-700 \mathrm{~nm}$ in steps of $10 \mathrm{~nm}$. First of all, as designed, the sample does not scatter light to angles below $30^{\circ}$ and above $75^{\circ}$. As mentioned earlier, the design is optimized to have only the first diffraction orders at $\lambda \geq 650 \mathrm{~nm}$. This can be observed in the measured spectra (Figure 4a). For shorter wavelengths $\lambda \leq 650 \mathrm{~nm}$, second-order diffraction in reflection is noticeable at high angles. Furthermore, following the grating equation, the diffraction angle for each metagrating increases as the illumination wavelength increases. The measurement significantly reflects very similar features in the simulated angular reflectance spectra in Figure $4 \mathrm{~b}$.

Figure $4 \mathrm{c}$ shows the measured angular reflectance at $\lambda=650 \mathrm{~nm}$. The scattered part of the light is depicted in red, whereas the gray dashed line represents the ideal Lambertian cosine distribution. Experimentally, the angular reflectance follows the desired cosine law rather well for angles in the range $30^{\circ}-75^{\circ}$. The two high-reflection peaks at $45^{\circ}$ are the result of a nonideal design; they are also visible in simulations (see Figure $4 \mathrm{~d}$ and Table S1). The blue part of the 
a)

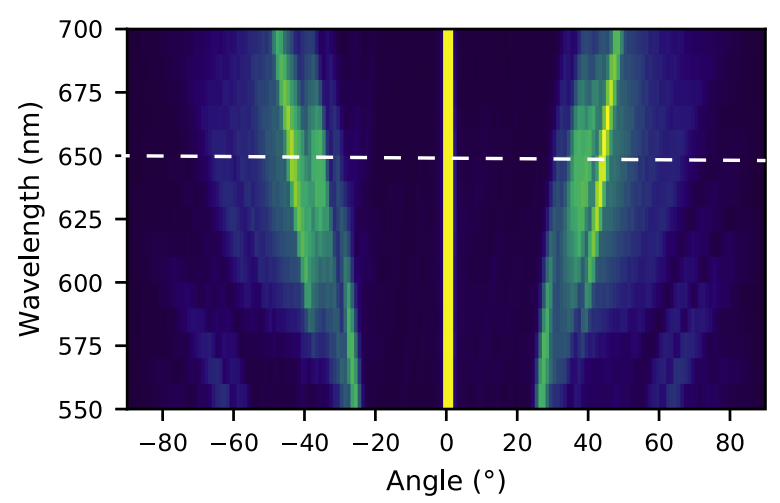

c)

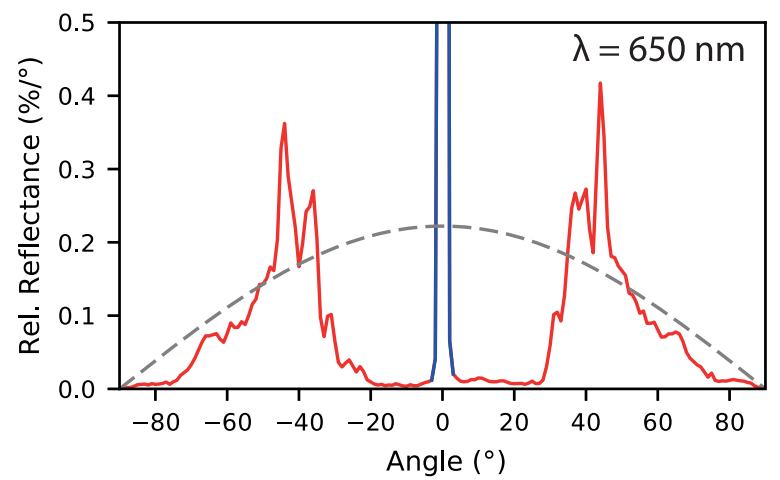

b)

\section{Simulation}

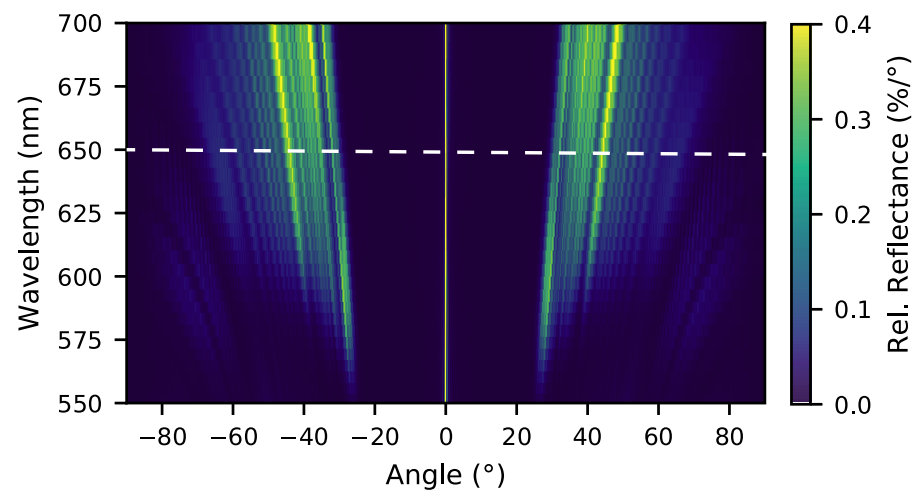

d)

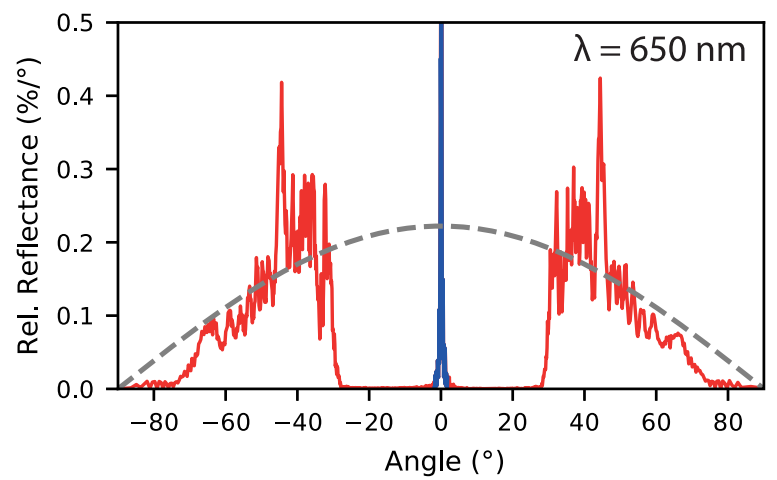

Figure 4. Angle-resolved reflectance. (a) Measured and (b) simulated angular reflectance of full metasurface. White dashed lines indicate cross section of the reflectance shown in parts $\mathrm{c}$ and d. (c) Angular reflectance at $\lambda=650 \mathrm{~nm}$ with specular (blue solid line) and diffracted (red solid line) contributions. The scattering profile of a perfect Lambertian scatterer is plotted as well (gray dashed line). (d) Simulated data as in part c.

angular reflectance represents the zeroth-order reflection and peaks at $6.8 \% / \mathrm{deg}$ and $3.0 \% / \mathrm{deg}$ in experiment and simulations, respectively (out of scale in Figure 4c,d). We observe a broader reflection range for the zeroth-order peak in the measurement compared to that in simulation, due to a difference in binning $\left(0.18^{\circ}\right.$ in simulation versus $1.0^{\circ}$ in measurement). Overall, we conclude that the experimental results follow the simulation results with good agreement.

3.3. Electrical Characterization. Next, we apply the transparent metagrating on a Si solar cell. The nanopatterned sapphire substrate was placed on top of a heterojunction silicon solar cell using immersion oil (Fluka 10976, $n=1.52$ ) (same setup as in ref 19). The cell features a textured front surface and a standard antireflection coating. We measured the external quantum efficiency (EQE) for three different cases: bare solar cell, cell with clean sapphire substrate, and cell with the fabricated metagrating sample (see Figure 5). With the sapphire substrate in place, we observe a similar trend as for the bare silicon solar cell, with slightly lower EQE over the whole spectrum. The reduction in EQE is due to reflection of light from the front surface of the sapphire, which is around $8 \%$ over the visible wavelength range. The EQE of the cell with the metagrating sample on top is lower compared to the EQE of the cell with sapphire, especially below $\lambda=800 \mathrm{~nm}$. A closer inspection of the spectrum shows a reduction of the EQE from $\lambda=600-800 \mathrm{~nm}$, which is due to the as-designed enhanced reflection shown in Figure 3a. The inset in Figure 5 shows a photo taken under $45^{\circ}$ of the sample placed on top of the solar cell. The bright red rooftop-like colored reflection is clearly visible.

In the lower-wavelength range, the EQE is reduced due to the higher absorption in the silicon nanowires. This could potentially be reduced by replacing the silicon with a material with a lower absorption in the visible range (e.g., $\mathrm{TiO}_{2}$ ). The measured short circuit current density for the bare silicon solar cell is $J_{\mathrm{sc}}=38.9 \mathrm{~mA} /$ $\mathrm{cm}^{2}$. Including the metagrating sample on top slightly reduces the

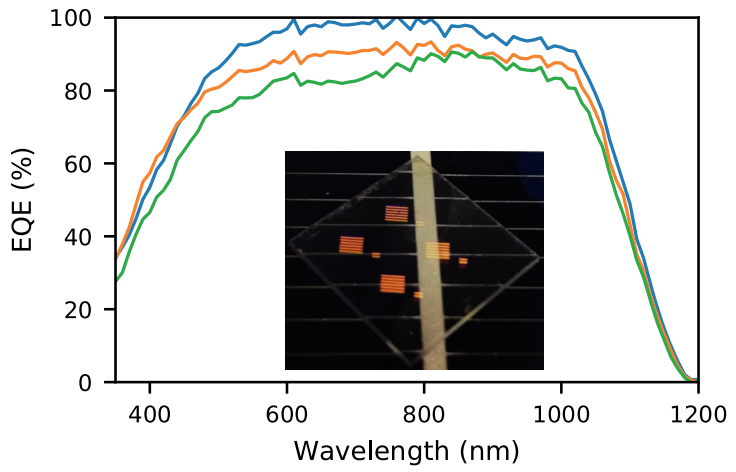

Figure 5. External quantum efficiency measurements. Measured external quantum efficiency of bare silicon heterojunction cell (blue line), with bare sapphire on top (orange line) and with metasurface (green line). Inset: Photograph (taken under $45^{\circ}$ ) of the sample attached to the silicon solar cell using immersion oil. The sample size is $12 \mathrm{~mm} \times 12 \mathrm{~mm}$. The four red patches are four metasurfaces with the same underlying design but fabricated with a slightly different dose during e-beam lithography. On the solar cell, silver contact fingers (horizontal gray lines) and a bus bar (vertical gray line) can be seen.

value to $J_{\mathrm{sc}}=34.0 \mathrm{~mA} / \mathrm{cm}^{2}$. This implies that to the first order the cell efficiency is reduced by relatively $13 \%$ due to the narrow-angle colored scattering metasurface.

\section{CONCLUSION}

In this work, we experimentally demonstrated spectral and angular control of scattered light using resonant metagratings 
for colored photovoltaics. Silicon nanowires were fabricated on a transparent substrate and resonantly scatter light around $\lambda=$ $650 \mathrm{~nm}$. The nanowires were placed in a supercell structure composed of multiple gratings with different pitches, creating a Lambertian scattering distribution with specular reflection that is nearly fully suppressed and efficient transmission outside the resonant scattering band. The supercell structure was designed to backscatter light within the $30-75^{\circ}$ range, and experiments and simulations are in very good agreement. The metagratings are integrated with a silicon heterojunction solar cell creating a bright red scattering appearance under a limited range of angles, as desired, and enable seamless integration with roof tile geometries. The external quantum efficiency is only reduced in the scattering spectral band, with a short circuit current drop of only $13 \%$. Future asymmetric designs ${ }^{24,36}$ may further enhance the efficiency by scattering light only to the ground and not to the sky. The nanopatterned geometries presented here can be readily fabricated and up-scaled to practical PV applications. The sapphire substrate can be replaced by solar panel module glass, and the crystalline Si Mie scatterers can be replaced by other high-index materials such as amorphous $\mathrm{Si}$ and $\mathrm{TiO}_{2}$, the latter potentially also via a sol-gel route. The SCIL technique ${ }^{37}$ used here is already being developed into a large-scale fabrication technique using a cassette-based wafer handling while a roll-to-roll soft imprint is also widely being developed. Furthermore, tailored spray coating techniques could also be employed to create arrays of nanoparticles.

\section{ASSOCIATED CONTENT}

\section{(s) Supporting Information}

The Supporting Information is available free of charge at https://pubs.acs.org/doi/10.1021/acsaem.0c00027.

Scattering spectrum of Si NW, metagrating efficiency, parameter overview, period dependence of metagrating reflectance, analysis of angle of incidence, and integrating sphere measurement setup (PDF)

\section{AUTHOR INFORMATION}

\section{Corresponding Author}

Verena Neder - Institute of Physics, University of Amsterdam, 1098 XH Amsterdam, The Netherlands; Center for Nanophotonics, NWO-Institute AMOLF, 1098 XG Amsterdam, The Netherlands; (1) orcid.org/0000-0003-0460391X; Email: v.neder@amolf.nl

\section{Authors}

Floris Uleman - Institute of Physics, University of Amsterdam, 1098 XH Amsterdam, The Netherlands; Center for Nanophotonics, NWO-Institute AMOLF, 1098 XG Amsterdam, The Netherlands

Andrea Cordaro - Institute of Physics, University of Amsterdam, 1098 XH Amsterdam, The Netherlands; Center for Nanophotonics, NWO-Institute AMOLF, 1098 XG Amsterdam, The Netherlands; $\odot$ orcid.org/0000-0003-30007943

Andrea Alù - Photonics Initiative, Advanced Science Research Center, City University of New York, New York, New York 10031, United States

Albert Polman - Center for Nanophotonics, NWO-Institute AMOLF, 1098 XG Amsterdam, The Netherlands; () orcid.org/0000-0002-0685-3886
Complete contact information is available at: https://pubs.acs.org/10.1021/acsaem.0c00027

\section{Notes}

The authors declare no competing financial interest.

\section{ACKNOWLEDGMENTS}

This work is part of the research program of AMOLF which is partly financed by the Dutch Research Council (NWO) and supported by the AFOSR MURI with Grant FA9550-17-10002 .

\section{REFERENCES}

(1) Heinstein, P.; Ballif, C.; Perret-Aebi, L.-E. Building Integrated Photovoltaics (BIPV): Review, Potentials, Barriers and Myths. Green 2013, 3 (2), 125-156.

(2) Schüler, A.; Roecker, C.; Scartezzini, J.-L.; Boudaden, J.; Videnovic, I. R.; Ho, R. S.-C.; Oelhafen, P. On the Feasibility of Colored Glazed Thermal Solar Collectors Based on Thin Film Interference Filters. Sol. Energy Mater. Sol. Cells 2004, 84 (1-4), 241254.

(3) Schüler, A.; Boudaden, J.; Oelhafen, P.; De Chambrier, E.; Roecker, C.; Scartezzini, J. L. Thin Film Multilayer Design Types for Colored Glazed Thermal Solar Collectors. Sol. Energy Mater. Sol. Cells 2005, 89 (2-3), 219-231.

(4) Li, M.; Zeng, L.; Chen, Y.; Zhuang, L.; Wang, X.; Shen, H. Realization of Colored Multicrystalline Silicon Solar Cells with $\mathrm{SiO} 2$ /SiN X:H Double Layer Antireflection Coatings. Int. J. Photoenergy 2013, 2013, 1-8.

(5) Soman, A.; Antony, A. Colored Solar Cells with Spectrally Selective Photonic Crystal Reflectors for Application in Building Integrated Photovoltaics. Sol. Energy 2019, 181, 1-8.

(6) Ji, C.; Zhang, Z.; Masuda, T.; Kudo, Y.; Guo, L. J. Vivid-Colored Silicon Solar Panels with High Efficiency and Non-Iridescent Appearance. Nanoscale Horizons 2019, 4 (4), 874-880.

(7) Pélisset, S.; Joly, M.; Chapuis, V.; Schueler, A.; Mertin, S.; HodyLe Caër, V.; Ballif, C.; Perret-Aebi, L.-E. Efficiency of Silicon ThinFilm Photovoltaic Modules with a Front Coloured Glass. Proceeding CISBAT Int. Conf.2011 2011, 37-42.

(8) Klampaftis, E.; Ross, D.; Richards, B. S. Color, Graphic Design and High Efficiency for Photovoltaic Modules. In IEEE 40th Photovoltaic Specialist Conference (PVSC); IEEE, 2014; pp 00250029. DOI: 10.1109/PVSC.2014.6925025.

(9) Jolissaint, N.; Hanbali, R.; Hadorn, J.-C.; Schüler, A. Colored Solar Façades for Buildings. Energy Procedia 2017, 122, 175-180.

(10) Masuda, T.; Kudo, Y.; Banerjee, D. Visually Attractive and High-Power-Retention Solar Modules by Coloring with Automotive Paints. Coatings 2018, 8 (8), 282.

(11) Pilliod, Mi.; Gougoussis, C.; Lefevre, O.; Laurin, M.; Liu, J. Obscuring, Color Matching, and Camouflaging Solar Panels. US Patent 20180122973, May 3, 2018.

(12) Peharz, G.; Berger, K.; Kubicek, B.; Aichinger, M.; Grobbauer, M.; Gratzer, J.; Nemitz, W.; Großschädl, B.; Auer, C.; Prietl, C.; Waldhauser, W.; Eder, G. C. Application of Plasmonic Coloring for Making Building Integrated PV Modules Comprising of Green Solar Cells. Renewable Energy 2017, 109, 542-550.

(13) Lee, K.-T.; Jang, J.-Y.; Zhang, J.; Yang, S.-M.; Park, S.; Park, H. J. Highly Efficient Colored Perovskite Solar Cells Integrated with Ultrathin Subwavelength Plasmonic Nanoresonators. Sci. Rep. 2017, 7 (1), 10640.

(14) Cho, E.-H.; Kim, H.-S.; Cheong, B.-H.; Oleg, P.; Xianyua, W.; Sohn, J.-S.; Ma, D.-J.; Choi, H.-Y.; Park, N.-C.; Park, Y.-P. TwoDimensional Photonic Crystal Color Filter Development. Opt. Express 2009, 17 (10), 8621.

(15) Cao, L.; Fan, P.; Barnard, E. S.; Brown, A. M.; Brongersma, M. L. Tuning the Color of Silicon Nanostructures. Nano Lett. 2010, 10 (7), 2649-2654. 
(16) Kanamori, Y.; Ozaki, T.; Hane, K. Reflection Color Filters of the Three Primary Colors with Wide Viewing Angles Using Common-Thickness Silicon Subwavelength Gratings. Opt. Express 2014, 22 (21), 25663.

(17) Proust, J.; Bedu, F.; Gallas, B.; Ozerov, I.; Bonod, N. AllDielectric Colored Metasurfaces with Silicon Mie Resonators. ACS Nano 2016, 10 (8), 7761-7767.

(18) Sun, S.; Zhou, Z.; Zhang, C.; Gao, Y.; Duan, Z.; Xiao, S.; Song, Q. All-Dielectric Full-Color Printing with TiO 2 Metasurfaces. ACS Nano 2017, 11 (5), 4445-4452.

(19) Neder, V.; Luxembourg, S. L.; Polman, A. Efficient Colored Silicon Solar Modules Using Integrated Resonant Dielectric Nanoscatterers. Appl. Phys. Lett. 2017, 111 (7), 073902.

(20) Neder, V.; Luxembourg, S. L.; Polman, A. Colored Solar Modules Using Integrated Pixelated Resonant Dielectric Nanoscatterer Arrays. In 33rd European Photovoltaic Solar Energy Conference and Exhibition; 2017; pp 34-37.

(21) Zhang, Y.; Chen, S.; Hu, D.; Xu, Y.; Wang, S.; Qin, F.; Cao, Y.; Guan, B.-O.; Miroshnichenko, A.; Gu, M.; Li, X. Coloring Solar Cells with Simultaneously High Efficiency by Low-Index Dielectric Nanoparticles. Nano Energy 2019, 62, 682-690.

(22) Ra'di, Y.; Sounas, D. L.; Alù, A. Metagratings: Beyond the Limits of Graded Metasurfaces for Wave Front Control. Phys. Rev. Lett. 2017, 119 (6), 067404.

(23) Epstein, A.; Rabinovich, O. Unveiling the Properties of Metagratings via a Detailed Analytical Model for Synthesis and Analysis. Phys. Rev. Appl. 2017, 8 (5), 054037.

(24) Khaidarov, E.; Hao, H.; Paniagua-Domínguez, R.; Yu, Y. F.; Fu, Y. H.; Valuckas, V.; Yap, S. L. K.; Toh, Y. T.; Ng, J. S. K.; Kuznetsov, A. I. Asymmetric Nanoantennas for Ultrahigh Angle Broadband Visible Light Bending. Nano Lett. 2017, 17 (10), 6267-6272.

(25) Paniagua-Domínguez, R.; Yu, Y. F.; Khaidarov, E.; Choi, S.; Leong, V.; Bakker, R. M.; Liang, X.; Fu, Y. H.; Valuckas, V.; Krivitsky, L. A.; Kuznetsov, A. I. A Metalens with a Near-Unity Numerical Aperture. Nano Lett. 2018, 18 (3), 2124-2132.

(26) Liu, W.; Miroshnichenko, A. E. Beam Steering with Dielectric Metalattices. ACS Photonics 2018, 5, 1733.

(27) Yang, J.; Sell, D.; Fan, J. A. Freeform Metagratings Based on Complex Light Scattering Dynamics for Extreme, High Efficiency Beam Steering. Ann. Phys. 2018, 530 (1), 1700302.

(28) Rabinovich, O.; Epstein, A. Analytical Design of Printed Circuit Board (PCB) Metagratings for Perfect Anomalous Reflection. IEEE Trans. Antennas Propag. 2018, 66 (8), 4086-4095.

(29) Neder, V.; Ra'di, Y.; Alù, A.; Polman, A. Combined Metagratings for Efficient Broad-Angle Scattering Metasurface. ACS Photonics 2019, 6 (4), 1010-1017.

(30) van de Groep, J.; Polman, A. Designing Dielectric Resonators on Substrates: Combining Magnetic and Electric Resonances. Opt. Express 2013, 21 (22), 26285.

(31) Kuznetsov, A. I.; Miroshnichenko, A. E.; Brongersma, M. L.; Kivshar, Y. S.; Luk'yanchuk, B. Optically Resonant Dielectric Nanostructures. Science 2016, 354 (6314), aag2472.

(32) Palik, E. D. Handbook of Optical Constants of Solids; Academic: New York, 1985.

(33) https://www.lumerical.com/products/.

(34) Taflove, A.; Hagness, S. C. Computational Electrodynamics: The Finite-Difference Time-Domain Method; Artech House Publishers, 2005.

(35) Cordaro, A.; van de Groep, J.; Raza, S.; Pecora, E. F.; Priolo, F.; Brongersma, M. L. Antireflection High-Index Metasurfaces Combining Mie and Fabry-Pérot Resonances. ACS Photonics 2019, 6 (2), 453-459.

(36) Chalabi, H.; Ra'di, Y.; Sounas, D. L.; Alù, A. Efficient Anomalous Reflection through Near-Field Interactions in Metasurfaces. Phys. Rev. B: Condens. Matter Mater. Phys. 2017, 96 (7), 075432.

(37) Verschuuren, M. A.; Megens, M.; Ni, Y.; Van Sprang, H.; Polman, A. Large Area Nanoimprint by Substrate Conformal Imprint Lithography (SCIL). Adv. Opt. Technol. 2017, 6 (3-4), 243-264. 